\title{
Experimental Evaluation Scheme of Ultra-wideband Human Body Transmission Model for Wireless Body Area Network
}

\author{
Aditep Chaisang and Sathaporn Promwong* \\ Faculty of Engineering, King Mongkut's Institute of Technology Ladkrabang, \\ 1 Soi Chalongkrung 1, Ladkrabang, Bangkok 10520, Thailand
}

(Received December 18, 2017; accepted September 11, 2018)

Keywords: UWB, UWB-HB, UWB-IR, WBAN, impulse radio

The body area network (BAN) working on IEEE 802.15.6 is a wireless short-range communication system that is smaller in coverage than the personal area network (PAN) IEEE802.15.3a. A wireless body area network (WBAN) is a wireless technology with a human body that is becoming a key part of short-range radio wave systems and wireless medical technology. The transfer function characteristics of the human body and the transmission loss due to the human body should be studied. Short-range radio wave applications with a human body are more complicated than additional uses. Therefore, realizing the distribution channel characteristics of the human body with ultra-wideband (UWB) technology is important. UWB technology has a very large bandwidth, low power, and low cost, which can be used in shortrange systems. In this paper, we present the characterization measurement data of the UWBHB channel transfer function based on the human body effect with and without human body in WBAN system. In this study, we maximize the signal-to-noise ratio (SNR) and contemplate the matched filter at the receiver. The outcomes are analyzed and evaluated on the basis of Friis' formula. The proposed method achieves very precise results and is convenient for the evaluation and design of the waveform of WBAN transmission in wireless medical applications and BAN systems, mainly focusing on the input waveform effect.

\section{Introduction}

Wireless body area network (WBAN) communication based on the wireless ultra-wideband (WUWB) technology has become a major topic for short-range communication systems with low-power and low-cost systems. ${ }^{(1,2)}$ The WUWB is dissimilar from additional impulse radio technologies. Instead of using the narrowband like other RF technologies, WUWB transmits low power spectral density (PSD) and tiny pulses in the range of its operating spectrum. The WUWB is an exclusive and new operation of a recently licensed frequency spectrum. The Federal Communication Committee (FCC) has regulated the occupied bandwidth equal to or more than $500 \mathrm{MHz}$ or a bandwidth fractional frequency equal to or more than 0.2 working and frequency spectrum for ultra-wideband (UWB) signal equal to $7.5 \mathrm{GHz}$, which varies from 3.1-10.6

*Corresponding author: e-mail: sathaporn.pr@kmitl.ac.th https://doi.org/10.18494/SAM.2018.1853 
GHz. ${ }^{(3)}$ The bandwidth of UWB can be written as

$$
B_{f}(f)=2 \frac{\left(f_{H}-f_{L}\right)}{f_{H}+f_{L}} \geq 0.2
$$

The low $f_{L}$ is the lowest frequency and $f_{H}$ is the highest frequency at $-10 \mathrm{~dB}$. The FCC stated that the PSD of the UWB signal should not be over $-41.3 \mathrm{dBm} / \mathrm{MHz}$ or over the FCC part 15 limits, which made the UWB signal to be considered as noise for other radio communication systems. Moreover, the UWB communication system can coexist without involvement with other wireless communication technologies. Therefore, the UWB impulse radio technology is a promising contender that may be used for commercial uses owing to its low budget and low power transmission within an indoor network such as wireless personal area networks (WPANs). ${ }^{(4)}$

In wireless communication in a narrowband, Friis' formula is expansively used to analyze the path loss in free space. ${ }^{(5)}$ Afterward, for WUWB communication, the complex form of Friss' formula has emerged to analyze the transmission waveform. ${ }^{(6-8)}$ The optimum WUWB receiver is used as the matched filter. ${ }^{(9-11)}$ To derive the signal-to-noise ratio (SNR) gain, the rectangular waveform is used in a WUWB free space channel, ${ }^{(12)}$ however, the discussion about the transmission channel measurement and WUWB antennas is insufficient.

WBAN has become the main short-range radio wave communication system. WBAN helps in the development of the health care industry and biological and information technologies. In the ideal term of WBAN, the wireless networks should have a working range away from the human body between 1-4 m. WBAN is categorized for applications such as medical and nonmedical uses. Factors such as reliability and power consumption are essential for WBAN uses in medical treatment. In the case of nonmedical uses, sound and image transmissions require a high-speed data transfer rate. Because it relies on the location of a device, the application of WBAN should be grouped as an implant and wearable device. For most medical treatments that can be categorized as an implant case, wireless amusement and entertainment applications can be categorized as a wearable device. ${ }^{(13-15)}$

In IEEE 802.15.6 TG, the standard of BAN has been described, including details about the OSI layer for BAN. To develop a communication link, the channel modeling is essential. The WBAN channel models are sorted as in-body, on-body, and off-body. Even so, a right model explains the WBAN channel characteristics but is still not available. In this point, it is not easy to design and evaluate the proper WUWB network.

In this research, we evaluated the UWB-HB transmission waveform for WBAN with Friis' formula that can be adapted for UWB impulse radio systems. However, in the evaluation of waveform distortion, the UWB-HB transfer function and a brief conclusion on the experimental study of the UWB-HB transmission model are considered in this paper. Besides, in this paper, we also use an extended formula of Friis, which is applied to the UWB-HB system model to evaluate the transfer function and measure the transmission transfer function with the vector network analyzer (VNA). The UWB-HB transmission waveform model is acceptable to be used in wireless medical research. 


\section{UWB-HB Transmission Model}

In this research, we consider the UWB-HB system link budget in free space evaluation. In a narrowband system, to calculate the link budget of the propagation loss in free space, Friis' formula usually is used as a suitable technique. ${ }^{(6)}$ Therefore, for the WUWB transmission system, it cannot be directly relevant to the formula that is represented in terms of the frequency function. Furthermore, because of the antenna frequency characteristics and the distortion of waveform, ${ }^{(7)}$ we had discussed and recommended the time-domain antenna effective length. One may treat the special cases of the steady UWB-HB gain and the steady aperture. In a narrowband line-of-sight (LOS) channel in the communication system, Friis' formula had been applied as

$$
G_{\text {Friis }}(f)=\frac{P_{r}(f)}{P_{t}(f)}=G_{f}(f) G_{r}(f) G_{t}(f)
$$

where $G_{t}(f)$ and $G_{r}(f)$ are the Tx and Rx gains, respectively.

$$
G_{f}(f)=\left(\frac{\lambda}{4 \pi d}\right)^{2}
$$

Here, $G_{f}(f)$ is the propagation gain in free space, $c$ is the velocity of light, $\lambda=\frac{c}{f}$ is the wavelength, and $f$ is frequency, and the separate distance between Tx and Rx antennas is shown in Fig. 1.

However, note that Eq. (2) is satisfied only at some precise frequency, and cannot immediately be applied to wireless UWB communication. Friis' formula is modified to analyze the waveform transfer function as well.

The impulse input is convolution and the pulse shaping at the transmitter side can express the input signal waveform $v_{i}(t)$. It is $h_{i}(t)$ as

$$
v_{i}(t)=\delta(t) \times h_{i}(t)
$$

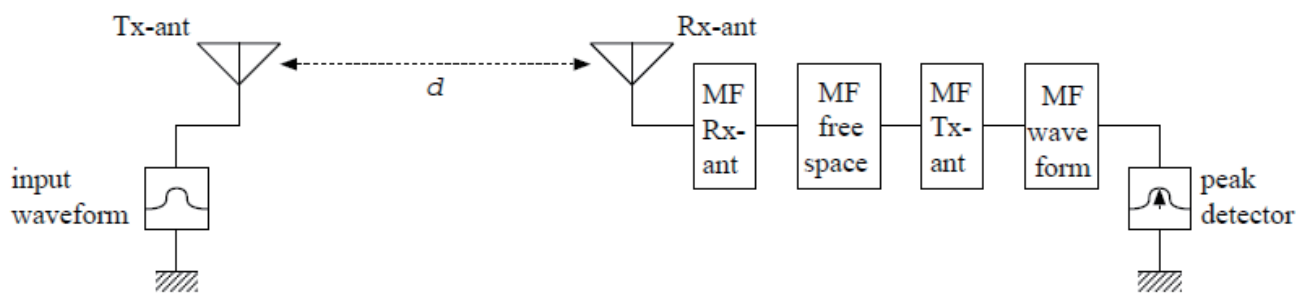

Fig. 1. UWB-HB system model for WBAN system. 
and

$$
\int_{-\infty}^{\infty} h_{i}^{2}(t) d t=\int_{-\infty}^{\infty}\left|H_{i}(f)\right|^{2} d f=1
$$

The UWB-HB channel transfer function of the receiver introduces the matched filter $H_{M F}(f)$ to maximize the SNR, as shown in Eq. (6).

$$
H_{M F}(f)=\frac{H_{e-F r i i s}^{*}(f)}{\sqrt{\int_{-\infty}^{\infty}\left|H_{e-F r i i s}^{*}\right|^{2} d f}} .
$$

In the isotropic case,

$$
H_{M F, I s o}(f)=\frac{H_{e-\text { Friis }}^{*}(f)}{\sqrt{\int_{-\infty}^{\infty}\left|H_{e-F r i s}^{*}\right|^{2} d f}},
$$

which fulfills the condition of the output noise power:

$$
\int_{-\infty}^{\infty}\left|H_{M F}(f)\right|^{2} d f=1
$$

In this illustration, the channel transfer functions of the received signal waveform are $H_{e-F r i i s}(f)$ and $h_{e-F r i i s}(t)$, and $E_{i}=1$, the waveform output. The output waveform by using a filter with matched $v_{M F}(t)$ is

$$
\begin{aligned}
v_{M F}(t) & =h_{e-F r i i s}(t) \times h_{M F}(t) \\
& =\frac{h_{e-F r i i s}(t) \times h_{M F}(-t)}{\sqrt{\int_{-\infty}^{\infty} h_{e-F r i i s}^{2}(t) d t}},
\end{aligned}
$$

and in the isotropic case,

$$
\begin{aligned}
v_{M F, I S o}(t) & =h_{e-F r i i s, I s o}(t) \times h_{M F, I s o}(t) \\
& =\frac{h_{e-F r i i s, I s o}(t) \times h_{M F, I s o}(-t)}{\sqrt{\int_{-\infty}^{\infty} h_{e-F r i i s, I s o}^{2}(t) d t}},
\end{aligned}
$$


in the matched filter output waveform $v_{M F}(t)$,

$$
\begin{aligned}
v_{M F}(f) & =H_{e-\text { Friis, Iso }}(f) H_{M F}(f) \\
& =\frac{\left|H_{e-F r i i s}(f)\right|^{2}}{\sqrt{\int_{-\infty}^{\infty}\left|H_{e-F r i i s}(f)\right|^{2} d f}}
\end{aligned}
$$

and the maximum SNR as

$$
\begin{aligned}
\max _{t} v_{M F}(f) & =\int_{-\infty}^{\infty} V_{M F}(f) d f \\
& =\sqrt{\int_{-\infty}^{\infty}\left|H_{e-F r i s}(f)\right|^{2} d f .}
\end{aligned}
$$

Equation (12) is Friis' formula in the UWB-HB complex. This includes the antenna characteristics, free space propagation loss, and input signal waveform. From Eq. (12), it is clear that the antenna gain in free space cannot be explained in terms of UWB-HB gain in Eq. (2). Alternatively, the waveform effect that includes the transmission gain can be acquired from Eq. (12). The normalization with $H_{I s o}(f)=1$ is evaluated for the reference isotropic antenna. The gain of UWB-HB transmission is defined as

$$
G_{U W B}=\frac{\max _{t} v_{M F}(t)}{\max _{t} v_{M F, I s o}(t)} .
$$

\section{Experimental Evaluation Scheme}

\subsection{Experimental system}

In this paper, the VNA was used to measure and record the result of transfer function. ${ }^{(16)}$ In addition, this transfer function is a product of UWB antenna gains that are measured from the antenna transmitter (Tx) and the antenna receiver ( $\mathrm{Rx})$ in cases without and with a human body, which is work done in an anechoic chamber room.

\subsection{UWB-HB transmission experimental setup}

The experiments conduced in this research are separated into two models, i.e., with and without a human body. According to Fig. 2, the antenna with Tx is placed on the front of the human body and the body front of the Rx antenna. The Tx and the Rx antennas are facing away 
from the human body. The model of measurement with the Tx and Rx antennas is placed on the human body. The Tx antenna is fixed and then the Rx antenna is rotated from 0 to 360 degree parts of the human body.

\subsection{Transmitted signal waveform of UWB-HB system}

In this study, the transmitted signal waveform communicates with $\mathrm{FCC}$ regulation $\mathrm{UWB}^{(3)}$ from 3.1-10.6 GHz. The bandwidth and middle frequency bandwidth are $f_{b}=7.5 \mathrm{GHz}$ and $f_{0}=6.85$ $\mathrm{GHz}$, respectively. The transmission waveform is assumed by using an ASK single pulse with the frequency carrier $f_{0}$. To satisfy the required bandwidth of $f_{b}$, the pulse length was set to be $2 / f_{b}$. Then, the Nyquist roll-off filter was used to limit the signal with the roll-off filter $\alpha=0$ in the distribution with the Gaussian model $\left(f_{0}-\frac{f_{b}}{2}, f_{0}+\frac{f_{b}}{2}\right)$. Figure 3 shows the waveform of the transmitted impulse of the UWB-HB system. The antenna transfer function was used to simulate the pulse waveform transmission.

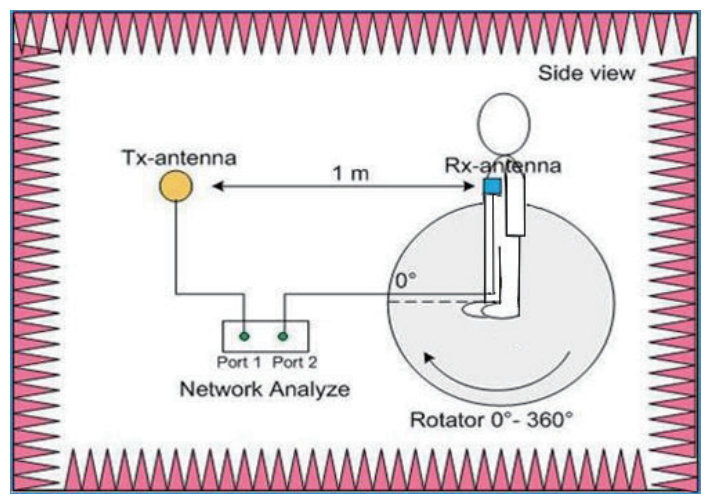

Fig. 2. (Color online) Experiment setup and UWB-HB antenna orientation.

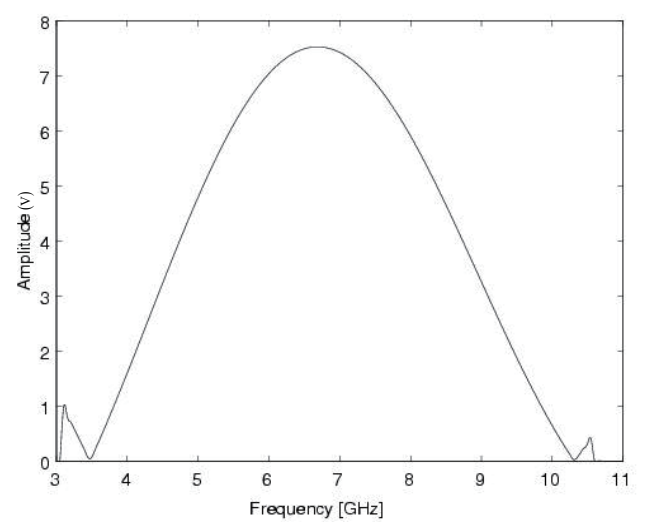

(a)

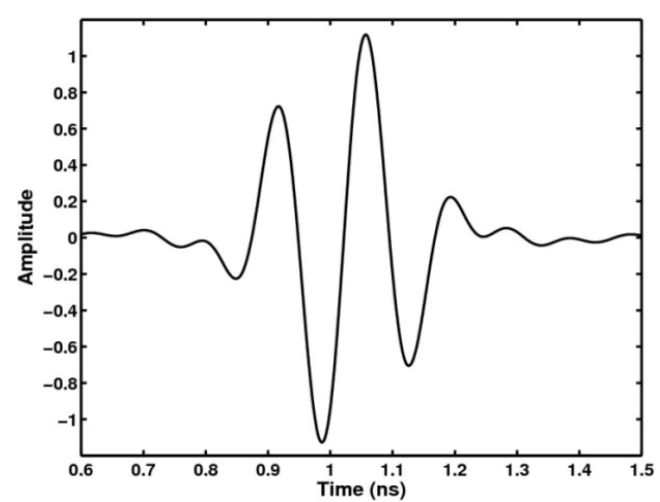

(b)

Fig. 3. Transmitted signal waveform of UWB-HB communication: (a) spectrum and (b) waveform. 


\subsection{Parameters of experiments}

Table 1 shows the list of important parameters for the experiments. Note that the cable connectors that are connected to the antennas had been calibrated. Hence, the measured results include all the characteristics of the antennas.

\section{Results and Discussion}

The experimental result of graphical compilation is described in this section. Figures 4 and 5 show the magnitude and phase of the UWB-HB channel transfer function in the case

Table 1

Parameters of experiments.

\begin{tabular}{lc}
\hline Parameters & Value \\
\hline Operating frequency span & $3-11 \mathrm{GHz}$ \\
Point of frequency & 1601 points \\
Type of Tx antenna & Biconical \\
Type of Rx antenna & Meander line \\
Rx antenna orientation & 0 to 360 degrees \\
Rotate step & 5 degrees \\
\hline
\end{tabular}

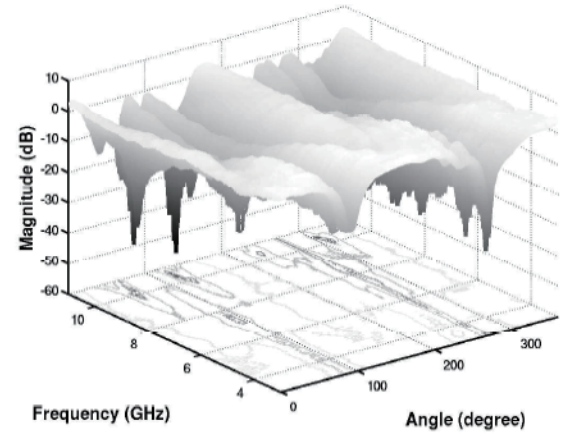

(a)

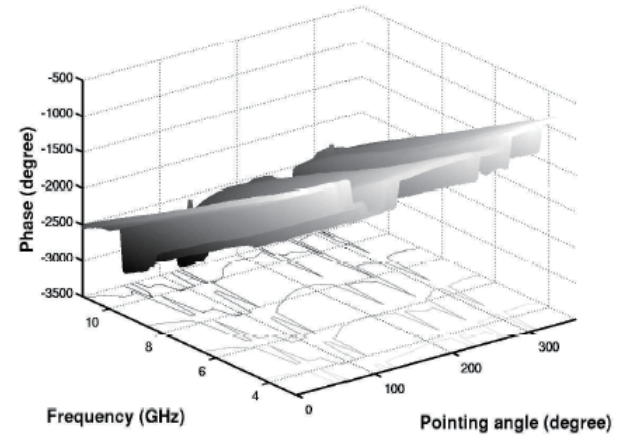

(b)

Fig. 4. UWB-HB transfer function without human body case: (a) magnitude and (b) phase.

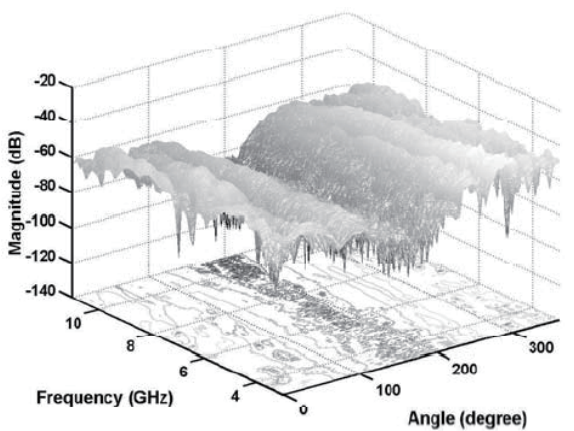

(a)

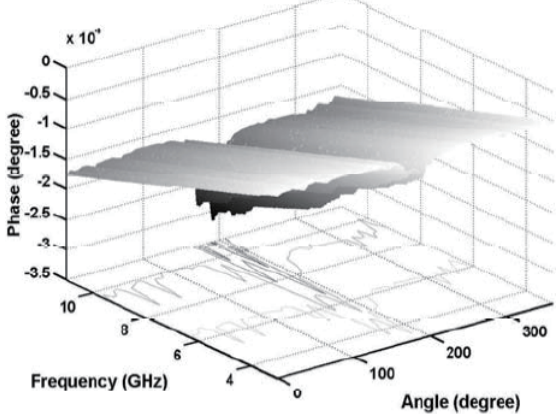

(b)

Fig. 5. UWB-HB transfer function with human body case: (a) magnitude and (b) phase. 
without and with a human body, respectively. In particular, at each pointing angle, the channel characteristic of the waveform transfer function is presented. In Fig. 4(a), the magnitude changes owing to the effect of the antenna characteristic. The phase is linear for the UWBHB transfer function in Fig. 4(b). In Fig. 5(a), the magnitude is affected by the human body shadowing around 180 degrees. The phase is mostly linear except the angle affected by the human body shadowing, as shown in Fig. 5(b).

Figure 6 shows the result of power delay profiles, which are measured in the cases without and with a human body. The power delay particularly explains the frequency characteristics of the delay spread and transfer functions, the shadowing effects due to the human body into the UWB-HB antenna transfer function, and the waveform level at all pointing angles due to the distance from the antenna. In Fig. 6 (a), there are null and small power delay profiles at 90 and 270 degrees, respectively, because it is the edge of the antenna at these angles. For Fig. 6(b), the power delay profile is small and null from 135 to 225 degrees, which have the human body shadowing effect.

Figure 7 shows the UWB-HB transmission gain in the case without and with a human body. In the case without a human body, the delay characteristic value is slightly changed. The signal is higher, because multipath fading occurs in the case with a human body. In

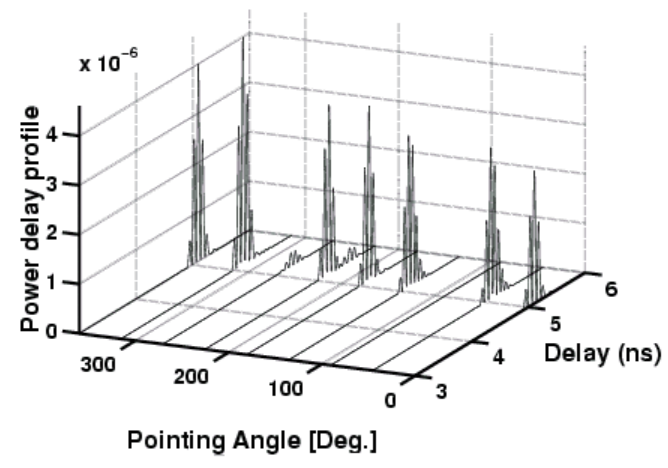

(a)

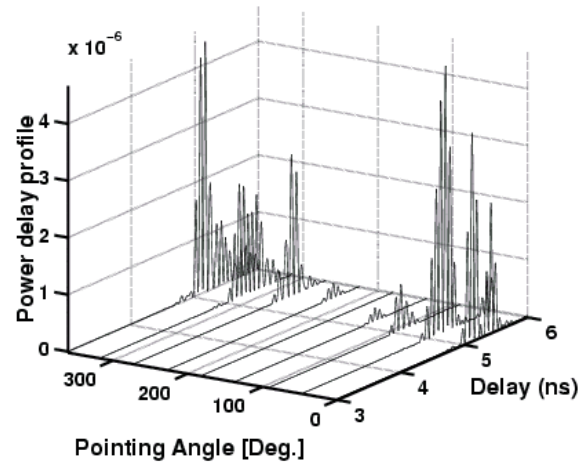

(b)

Fig. 6. Delay characteristic of the UWB-HB waveform (a) without body and (b) with body.

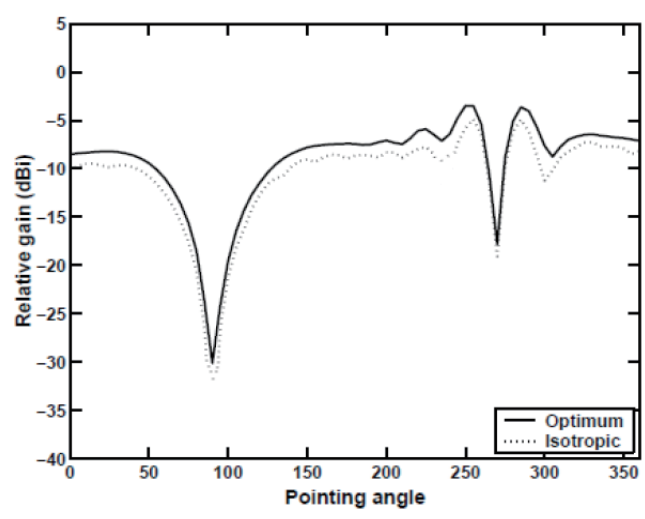

(a)

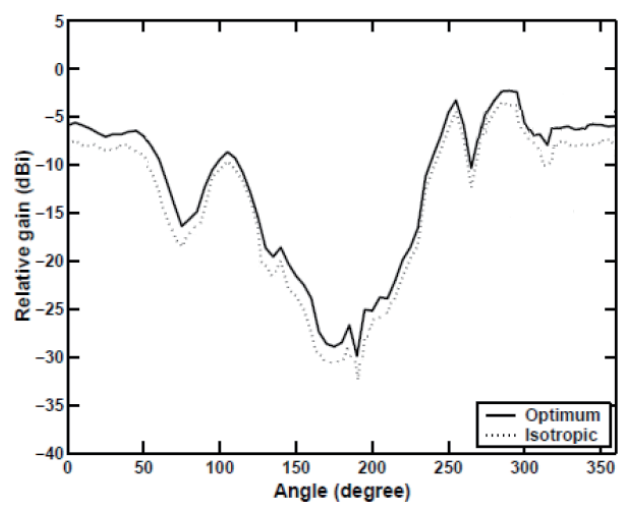

(b)

Fig. 7. UWB-HB transmission gain: (a) without body and (b) with body. 
Fig. 7(a), the transmission in the case without a human body is null at 90 and 270 degrees because of the antenna characteristic. In Fig. 7(b), the transmission gain is null at 180 degrees due to the shadowing effect from the human body. In this research, the optimum matched filter is introduced as the receiver and the result in Fig. 7 shows the comparison between the optimum matched filter and the isotropic matched filter, in which the difference in the average transmission gain is less than $2 \mathrm{~dB}$, meaning that the optimum matched filter can be used to evaluate the UWB-HB waveform transfer function.

\section{Conclusion}

In this work, we evaluated the UWB-HB waveform transfer function model for WBAN based on measurement data and the analysis using the complex form with the extended formula of Friis to consider the antenna performance and transmitted waveform. The matched filter is introduced to maximize the gain of SNR at the receiver side to obtain the best performance in WBAN communication, because the characteristic of the matched filter can increase the gain and there is no delay.

In the experimental system, we consider to use VNA to measure and record with a biconical antenna as Tx-antenna and a meander line as Rx-antenna. In the results, we evaluate the UWBHB channel transfer function and the UWB-HB transmission gain under two conditions: without a human body and with a human body. The result is good to be based on the modified Friis' formula and the matched filter. The UWB-HB transmission gain and waveform distortion due to the human body effect at $0,180,270$, and 360 degrees are known. The characteristics of UWB transmission impulse radio with the human body effect with this definition may be useful. In the waveform transmission, the performance is evaluated in terms of the UWBHB channel transfer function and transmission gain for wireless BAN. In this research, it is a special case to study the theory for evaluating the UWB-HB at the best scenario. In future work, since the matched filter is difficult to implement in real practice, other receiver templates such as correlators can be considered.

\section{Acknowledgments}

The authors would like to thank Mr. Thanadol Tiengthong of KMITL for his help in the review of this paper and experiments and Prof. Jun-ichi Takada of the Graduate School of Science and Engineering, Tokyo Institute of Technology, Japan, for letting us use their meander line antenna.

\section{References}

1 P. Pagani: Ultra-Wide Band Radio Propagation Channels (Wiley, Hoboken, 2008) pp. 21-23.

2 I. Oppermann, M. Hamalainen, and J. Iinatti: UWB Theory and Applications (Wiley, Chichester, 2004) p. 3.

3 Federal Communications Commission: Revision of Part 15 of the Commission's Rules Regarding UWB Transmission Systems, First Report (FCC, Apr. 2002).

4 J. Misic and V. Misic: Wireless Personal Area Network: Performance, Interconnection and Security with IEEE 802.15.4 (Wiley, Chichester, 2008) pp. 3-12. 
5 H. T. Friis: Proc. 1946 IRE (IRE, 1946) 254-256.

6 J. Takada, S. Promwong, and W. Hachitani: Technical Report of IEICE WBS2003-8. NW2003-20 (IEICE, 2003) 45-49.

7 S. Promwong, W. Hachitani, and J. Takada: Technical Meeting on Instrumentation and Measurement IM-0335 (IEE, 2003) 37-41.

8 S. Promwong, W. Hachitani, J. Takada, P. Supanakoon, and P. Tangtisanon: 3rd Int. Symp. Communications and Information Technology (ISCIT) 1 (2003) 467-470.

9 X. Xu and R. M. Narayanan: 2003 IEEE Int. Geoscience and Remote Sensing Symp. (IGARSS) 7 (2003) $4525-4527$.

10 S. Promwong, J. Takada, P. Supanakoon, and P. Tangtisanon: 2004 Int. Symp. Antenna and Propagation (ISAP, 2004) 97-100.

11 S. Promwong, J. Takada, P. Supanakoon, and P. Tangtisanon: IEEE TENCON 2004 on Analog and Digital Techniques in Electrical Engineering (IEEE TENCON, 2004) 125-128.

12 M. R. Yuce: Ultra-wideband and $60 \mathrm{GHz}$ Communications for Biomedical Applications (Springer, New York, 2014) pp. 3-20.

13 K. M. S. Thotahewa, J. M. Redoute, and M. R. Yuce: Ultra Wideband Wireless Body Area Network (Springer, New York, 2014).

14 J.-M. Choi, H.-J. Kang, and Y.-S. Choi: Proc. FGCN '08 (IEEE, 2008) 263-266.

15 S. Promwong, J. Takada, P. Supanakoon, and P. Tangtisanon: 2004 Int. Symp. Antenna and Propagation (ISAP, 2004) 89-92.

\section{About the Authors}

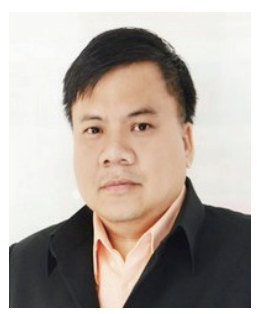

Aditep Chaisang received his M.E. degree in applied physics and B.S. degree in telecommunication. He joined the Department of Information and Communication Engineering, Faculty of Engineering, Rambhaibanni Rajapat University. He is now pursuing a doctoral degree at the Faculty of Engineering, KMITL. His research interests are in the areas of wireless body area network, antenna and radio wave propagation, and UWB systems.

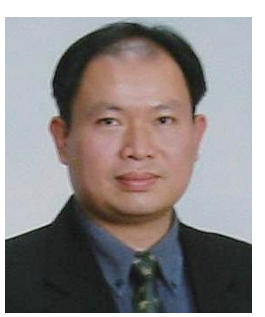

Sathaporn Promwong received his Ph.D. degree in communications and integrated systems from Tokyo Institute of Technology, Tokyo, Japan, his M.E. degree in electrical engineering, and his B.Ind.Tech. degree in electronic technology from KMITL, Bangkok, Thailand, in 2009, 1999, and 1994, respectively. $\mathrm{He}$ is a lecturer in the Department of Telecommunication Engineering, Faculty of Engineering, KMITL and a member of IEEE, IEICE, and ECTI. His research interests are in the areas of radio wave propagation, UWB technology, and antennas. 